\title{
Elucidating the Nature of Deregulated Semantic Cognition in Semantic Aphasia: Evidence for the Roles of Prefrontal and Temporo-parietal Cortices
}

\author{
Krist A. Noonan ${ }^{1}$, Elizabeth Jefferies ${ }^{2}$, Faye Corbett ${ }^{1}$, \\ and Matthew A. Lambon Ralph ${ }^{1}$
}

\begin{abstract}
Semantic cognition-semantically driven verbal and nonverbal behavior - is composed of at least two interactive principal components: conceptual representations and executive control processes that regulate and shape activation within the semantic system. Previous studies indicate that semantic dementia follows from a progressive yet specific degradation of conceptual knowledge. In contrast, multimodal semantic impairment in aphasic patients (semantic aphasia [SA]) reflects damage to the control component of semantic cognition [Jefferies, E., \& Lambon Ralph, M. A. Semantic impairment in stroke aphasia versus semantic dementia: A case-series comparison. Brain, 129, 2132-2147, 2006]. The purpose of the present study was to examine the nature of the semantic control deficits in SA in detail for the first time. Seven patients with SA were tested on four comprehension
\end{abstract}

\section{INTRODUCTION}

Semantic cognition encompasses the processes and representations that underpin our understanding of the meaning of words, objects, people, and facts. It provides the basis for our ability to make judgments about our environment and act in a coherent goal-directed manner with regard to the meaning rather than superficial characteristics of words, objects, etc. (Lambon Ralph \& Patterson, 2008). Disturbances of semantic cognition can reflect at least three types of impairment. First, the semantic representations themselves can be damaged (e.g., as in semantic dementia [SD]; Patterson, Nestor, \& Rogers, 2007; Jefferies \& Lambon Ralph, 2006; Rogers et al., 2004). Secondly, access to conceptual representations from a particular modality may be affected following damage to a specific sensory input (e.g., pure word deafness or visual agnosia). Finally, the executive regulation of semantic activity can be disrupted, as documented recently in aphasic patients with multimodal semantic impairment following stroke (Samson, Connolly, \& Humphreys, 2007; Jefferies \& Lambon Ralph, 2006). For

${ }^{1}$ University of Manchester, UK, ${ }^{2}$ University of York, UK and naming tasks that directly manipulated the requirement for executive control in different ways. In line with many theories of cognitive control, the SA patients demonstrated three core features of impaired control: (i) they exhibited poor on-line manipulation and exploration of semantic knowledge; (ii) they exhibited poor inhibition of strongly associated distractors; and (iii) they exhibited reduced ability to focus on or augment less dominant aspects of semantic information, although the knowledge itself remained and could be successfully cued by external constraints provided by the examiner. Our findings are consistent with the notion that the anterior temporal lobes are crucial for conceptual knowledge whereas the left prefrontal and temporoparietal cortices, damaged in patients with SA, play a critical role in regulating semantic activation in a task-appropriate fashion.

ease of reference, we will refer to this deficit as semantic aphasia (SA).

Research on semantic cognition has largely focused on the representational structure of semantic knowledge (Rogers et al., 2004; Caramazza \& Shelton, 1998; Warrington \& Shallice, 1984). The processes that support the selective, task-orientated application of conceptual knowledge have received less attention (Koenig \& Grossman, 2007; Jefferies \& Lambon Ralph, 2006). Nevertheless, these processes are critical to semantic cognition in all modalities: We store a wealth of information about the meanings of words/objects but frequently only a subset of this knowledge is required for a task-indeed, other aspects of knowledge may actually be inappropriate and unhelpful. For example, playing a piano requires information about fine movements of the fingers to be retrieved, yet moving a piano across a room requires very different actions (Saffran, 2000). Similarly, in tests of semantic association, such as the Pyramids and Palm Trees test (Howard \& Patterson, 1992), it is necessary to focus on the relevant relationship in each trial and reject other possible associations (e.g., to know that the pyramid goes with the palm tree not the pine tree, one must understand that the attribute "found in deserts" is relevant, whereas the shape or category of the objects is 
not). Even more straightforward tasks like word-picture matching and picture naming require semantic activation to be channeled toward the target and away from semantically related competitors. In picture naming and speech production, the speaker also needs to select the appropriate label (e.g., animal, pet, dog, springer spaniel, or "Oliver") to convey the correct level of semantic specificity for a given concept (known in speech production research as the hypernym problem; Levelt, 1992). In addition, the critical aspects of meaning can also change for the same concept over time, not only in language but also in nonverbal behavior such as object use. Imagine, for example, the radically different uses of the same knife in the task of making a cheese and chutney sandwich: packet opening, butter spreading, bread cutting, cheese slicing, chutney scooping, etc., all require different, specific aspects of the knife's properties (and ways of holding and manipulating it) to be brought to the fore, one by one, whereas the most commonly listed property of cutting has to be inhibited more often than not. Indeed, in the case of scooping, the canonical function of the knife has to be disregarded altogether and replaced by a substituted function in place of another object (spoon). In conclusion, the ability to regulate and to shape conceptual information in all expressive and receptive modalities is critical to any adequate account of semantic cognition.

The distinction between semantic representations and control processes helps to resolve a puzzle highlighted by a comparison of different, semantically impaired patient groups (i.e., patients who fail both verbal and nonverbal semantic tasks). Patients with SD show a progressive and specific impairment of semantic memory, which is frequency graded and affects the comprehension of stimuli presented in every modality, including spoken and written words, pictures, environmental sounds, and smells (Luzzi et al., 2007; Coccia, Bartolini, Luzzi, Provinciali, \& Lambon Ralph, 2004; Bozeat, Lambon Ralph, Patterson, Garrard, \& Hodges, 2000). SD results from circumscribed atrophy of the anterior temporal lobes (ATLs) bilaterally, suggesting that this brain region underpins our store of amodal semantic knowledge (Nestor, Fryer, \& Hodges, 2006; Rogers et al., 2004; Mummery et al., 2000). In contrast, multimodal semantic impairment in aphasia following stroke (SA) is associated with left hemisphere lesions to either $\mathrm{pFC}$ (especially left inferior frontal gyrus; BA 44, 45, 47) or posterior temporal/inferior parietal areas (e.g., BA 37, 20-22, 39/40), referred to here as TPJ. Hart and Gordon (1990) found that comprehension problems were consistently associated with damage to temporal and parietal areas (i.e., BA 37, 39, 40). Chertkow, Bub, Deaudon, and Whitehead (1997) reported similar findings for five semantically impaired patients who all showed damage to posterior inferior temporal areas (i.e., BA 37, 22, 21). Interestingly, evidence from transcortical sensory aphasia - a disorder characterized by poor comprehension in the context of fluent speech and preserved repetition-highlights that multimodal semantic impairment following stroke can result from isolated lesions to either the pFC (i.e., BA 44, $45,47)$ or the posterior temporal/inferior parietal areas (i.e., BA 37, 39, etc.) with few differences in the cognitive profiles of these two patient groups (Berthier, 2001).

Stroke rarely produces lesions of the ATL (and almost never results in bilateral ATL infarcts). This may be for two reasons: (i) although the exact arterial distribution varies from individual to individual, the ATL often has a double blood supply (the anterior temporal cortical artery of the middle cerebral artery and the anterior temporal branch of the distal posterior cerebral artery; Borden, 2006; Conn, 2003); and (ii) the anterior temporal cortical artery branches below the main trifurcation of the MCA and thus may be less vulnerable to emboli. As a consequence of this low stroke rate, this region is not considered in stroke-based neurological models of comprehension/semantic memory, whereas left TPJ and pFC are thought to be the critical neural substrates instead (Wise, 2003).

Clearly, all three of these brain regions (ATL, pFC, and TPJ) contribute to semantic cognition, but further research is needed to elucidate their precise roles. Functional neuroimaging studies of semantic cognition have largely focused on the contribution of left inferior frontal regions to semantic selection and controlled retrieval (Badre \& Wagner, 2002; Wagner, Pare-Blagoev, Clark, \& Poldrack, 2001; Thompson-Schill, D'Esposito, Aguirre, \& Farah, 1997; Demb et al., 1995). However, these studies have frequently observed posterior temporo-parietal activations that are also sensitive to the manipulation of semantic control demands (Badre, Poldrack, Pare-Blagoev, Insler, \& Wagner, 2005; Wagner et al., 2001; ThompsonSchill et al., 1997). In addition, coupled activation of $\mathrm{pFC}$ and posterior temporal areas has been observed across a range of semantic tasks that require the contextually appropriate activation of specific features of conceptual knowledge. For example, Rodd, Davis, and Johnsrude (2005) showed that judging whether a word was related to a preceding sentence required the activation of both frontal and posterior temporal areas only when the sentence contained many ambiguous words with competing interpretations (e.g., does "battle" go "the shell was fired toward the tank"?). Similarly, studies of ambiguity resolutions, using both homonyms and metaphors, indicate that when less common meanings need to be accessed-in the face of competition from the item's more dominant meaningpFC and posterior temporal cortex work in tandem to resolve conflict (Bedny, McGill, \& Thompson-Schill, 2008; Gennari, MacDonald, Postle, \& Seidenberg, 2007; Zempleni, Renken, Hoeks, Hoogduin, \& Stowe, 2007; Lee \& Dapretto, 2006). Beyond the temporal lobe, activation of inferior parietal structures is also commonly seen in semantic tasks (Vigneau et al., 2006). Once more, the evidence points toward a semantic control function for these areas. Cristescu, Devlin, and Nobre (2006) showed that inferior parietal cortex was engaged along with posterior temporal and 
inferior frontal areas when participants orientated attention toward semantic categories. Similarly, switching between clusters-groups of conceptually similar items - in semantic fluency has been associated with inferior parietal activation as well as inferior frontal cortex, suggesting that both areas are involved in high-level control (Hirshorn \& ThompsonSchill, 2006). The importance of parietal cortex in regulatory control has recently been investigated by Nagel, Schumacher, Goebel, and D'Esposito (2008) using both semantic and nonsemantic selection tasks. Their results showed that parietal cortex was activated, along with dissociable regions of $\mathrm{pFC}$, for both semantic and nonsemantic selection tasks. This suggests that parietal cortex contributes toward domain-general as well as semantic control. With this evidence in mind, the multimodal semantic deficits seen in patients with stroke aphasia following $\mathrm{pFC/}$ TPJ lesions might result from an inability to shape and regulate activation within the semantic system.

In a recent case-series study, Jefferies and Lambon Ralph (2006) directly compared SD and SA patients on a battery of semantic tests. The SD and the SA patients showed a similar degree of impairment on the same range of verbal and nonverbal semantic tasks despite their very different areas of brain damage (bilateral ATL in SD vs. left pFC and TPJ in SA). However, the nature of the semantic impairment was qualitatively different in the two groups consistent with the view that SD reflects degraded knowledge whereas SA results from deregulated semantic control. The SD patients were highly consistent across different semantic tasks: Patients who retained knowledge of an item in one task were typically able to demonstrate this knowledge in all other tasks. In contrast, patients with SA showed significant correlations/consistency only between different versions of the same semantic task (e.g., judgments of semantic association for words and pictures). Strikingly, they were often unable to retrieve information about concepts that they had understood in other tasks with different semantic control demands (e.g., judgments of semantic association vs. word-picture matching). Moreover, performance in the SD group showed a profound effect of item frequency/familiarity; in contrast, the SA patients showed little influence of this variable. Instead, their ability to make judgments of semantic association was predicted by how readily the relevant associative dimension could be discerned and competitors rejected. The patients' errors in picture naming provided further insight into the nature of the underlying semantic disorder in SD and SA. The SD patients made frequent coordinate and superordinate semantic errors (such as saying "dog" or "animal" for squirrel). The SA patients additionally made associative errors (e.g., producing the response "nuts" for squirrel); these responses were virtually never seen in the SD group. These errors reveal that the SA patients retained a surprising amount of knowledge about unnamed targets (to be able to generate such errors) and suggest that their difficulty lay in directing activation toward the correct name and away from irrelevant prepotent associations. In line with this, the SA patients showed a greater benefit than the SD patients of phonemic cues in picture naming. These cues provided an additional external constraint on semantic activation, reducing the need for internally generated control (Jefferies, Patterson, \& Lambon Ralph, 2008). Furthermore, the SA patients showed refractory effects in naming and word-picture matching (Jefferies, Baker, Doran, \& Lambon Ralph, 2007): Their performance declined when a small set of semantically related items was repeated, resulting in a buildup of interference (Gotts \& Plaut, 2002; Warrington \& Cipolotti, 1996; Warrington \& McCarthy, 1983). Therefore, semantic impairment in SA does not follow the pattern expected for disorders of semantic representation but is better characterized as a disruption to the executive processes that regulate semantic activity.

The study of Jefferies and Lambon Ralph (2006) clearly indicates that the multimodal semantic impairment in SA is qualitatively different from that in SD and is consistent with a semantic control deficit. The study, however, did not investigate the nature of the control deficits in the SA patients because we did not experimentally manipulate the nature and the degree of the semantic control demands of the tasks. In the current study, seven patients with SA were tested on four semantic tasks that were specifically designed to vary the requirement for semantic control in a variety of different ways. We explored the integrity of three aspects of control: (i) on-line manipulation and exploration of semantic knowledge; (ii) inhibition of strongly associated distractors; and (iii) the ability to focus on or augment less dominant aspects of semantic information. The current study also provides a further explicit test of the hypothesis that multimodal semantic impairment in stroke aphasia reflects an impairment of semantic control (rather than a loss of semantic knowledge per se). As noted above, the patients had lesions affecting left prefrontal and temporo-parietal cortex-areas that have been implicated in the executive control of semantic activation by functional neuroimaging studies of healthy volunteers. By examining whether lesions in these areas disrupt semantic control, this study explores the degree of convergence between neuropsychology and functional neuroimaging studies.

\section{PARTICIPANTS}

\section{Patients}

Seven of the 10 SA patients studied by Jefferies and Lambon Ralph (2006) took part in this study. Patients were selected to show multimodal semantic deficits affecting the comprehension of both words and pictures (see Table 1). All of the patients had chronic impairments resulting from a cerebrovascular accident (CVA) at least 1 year before testing. Four of the patients had transcortical sensory aphasia, one showed a mixed transcortical pattern, one was a conduction aphasic, and the last was 
Table 1. Background Neuropsychology

\begin{tabular}{|c|c|c|c|c|c|c|c|c|c|}
\hline & $\operatorname{Max}$ & Normal Cutoff & $S C$ & $P G$ & $N Y$ & $B B$ & $K A$ & $M E$ & $L S$ \\
\hline \multicolumn{10}{|l|}{ Semantic Tests } \\
\hline Composite semantic score & & & 1.56 & 0.67 & 0.41 & -0.13 & -0.45 & -0.91 & -2.10 \\
\hline Word-picture matching & 64 & 62 & $59^{\mathrm{a}}$ & $58^{\mathrm{a}}$ & $60^{\mathrm{a}}$ & $54^{\mathrm{a}}$ & $26^{\mathrm{a}}$ & $50^{\mathrm{a}}$ & $37^{\mathrm{a}}$ \\
\hline Picture naming & 64 & 59 & $28^{\mathrm{a}}$ & $46^{\mathrm{a}}$ & $55^{\mathrm{a}}$ & $10^{\mathrm{a}}$ & $0^{\mathrm{a}}$ & $5^{\mathrm{a}}$ & $5^{a}$ \\
\hline CCT pictures & 64 & 51 & $46^{\mathrm{a}}$ & $44^{\mathrm{a}}$ & $36^{\mathrm{a}}$ & $38^{\mathrm{a}}$ & $46^{\mathrm{a}}$ & $13^{\mathrm{a}}$ & $16^{\mathrm{a}}$ \\
\hline CCT words & 64 & 56 & 56 & $40^{\mathrm{a}}$ & $39^{\mathrm{a}}$ & $30^{\mathrm{a}}$ & $36^{\mathrm{a}}$ & $34^{\mathrm{a}}$ & $16^{\mathrm{a}}$ \\
\hline \multicolumn{10}{|c|}{ Attention, Executive, and Visuospatial Tests } \\
\hline Digit span forward & - & 5 & 6 & 6 & $3^{\mathrm{a}}$ & 5 & $0^{\mathrm{a}}$ & 6 & $4^{\mathrm{a}}$ \\
\hline Digit span backward & - & 2 & 2 & 2 & 2 & $0^{\mathrm{a}}$ & NT & 3 & $1^{\mathrm{a}}$ \\
\hline VOSP screening & 20 & 15 & 20 & 20 & 19 & 20 & 20 & 19 & 18 \\
\hline VOSP position discrimination & 20 & 18 & $17^{\mathrm{a}}$ & 20 & 20 & 18 & 14 & $15^{\mathrm{a}}$ & $16^{\mathrm{a}}$ \\
\hline VOSP number location & 10 & 7 & $0^{\mathrm{a}}$ & 9 & 10 & 8 & 6 & $2^{\mathrm{a}}$ & 8 \\
\hline Brixton Spatial Anticipation Test & 55 & 28 & $25^{\mathrm{a}}$ & $26^{\mathrm{a}}$ & 34 & $23^{\mathrm{a}}$ & $6^{\mathrm{a}}$ & $11^{\mathrm{a}}$ & $14^{\mathrm{a}}$ \\
\hline TEA elevator counting (no distraction) & 7 & 6 & 7 & $3^{\mathrm{a}}$ & $3^{\mathrm{a}}$ & $4^{\mathrm{a}}$ & TA & 7 & $3^{\mathrm{a}}$ \\
\hline TEA elevator counting (distraction) & 10 & 3 & $1^{\mathrm{a}}$ & $0^{\mathrm{a}}$ & $2^{\mathrm{a}}$ & $0^{\mathrm{a}}$ & NT & 9 & $2^{\mathrm{a}}$ \\
\hline Hayling sentences A (errors) & 45 & - & 24 & 13 & 5 & 17 & NT & 3 & 19 \\
\hline Hayling sentences B (errors) & 45 & 18 & $26^{\mathrm{a}}$ & $18^{\mathrm{a}}$ & $23^{\mathrm{a}}$ & TA & NT & 15 & $38^{\mathrm{a}}$ \\
\hline Raven's (percentiles) & - & - & 50 & 50 & 50 & 50 & $5^{\mathrm{a}}$ & $<5^{\mathrm{a}}$ & 10 \\
\hline WCST (no. categories) & 6 & 1 & 6 & $0^{\mathrm{a}}$ & 2 & 1 & 1 & $0^{\mathrm{a}}$ & $0^{\mathrm{a}}$ \\
\hline
\end{tabular}

Patients are arranged in order of their composite semantic score derived from the four background tests of semantic processing. NT $=$ not tested due to very poor spoken output. TA = testing abandoned.

${ }^{a}$ Denotes impaired performance.

globally aphasic. Table 2 provides a breakdown of the aphasic and demographic profiles of the patients.

\section{Lesion Analyses}

For six of seven patients, CT/MRI scans were manually traced onto Damasio's standardized templates (Damasio \& Damasio, 1989). PG was excluded due to the unavailability of a previous CT scan and contraindications for further magnetic resonance scanning. Radiological report indicated a left frontal lesion but made no definitive statement about more posterior damage. Analyses of the Damasio plates for the remaining six patients confirmed left prefrontal lesions and/or infarcts affecting the left posterior temporal/inferior parietal cortices. Four of six patients had damage to both frontal and TPJ sites, whereas two of six had infarcts confined to TPJ. Table 3 presents a breakdown of the patients lesions relative to ROIs defined by previous functional neuroimaging and neuropsychological studies of semantic cognition (Vigneau et al., 2006; Wagner et al., 2001; Chertkow et al., 1997; Thompson-Schill et al.,
1997; Demb et al., 1995; Hart \& Gordon, 1990). Patients showed extensive damage to peri-sylvian language structures, with the highest co-occurrence of damage in BA 44 in the frontal lobe and BA 37 in the posterior temporal lobe. When white-matter disruption was considered, inferior parietal structures (BA 39/40) were also frequently implicated. The term TPJ will be used throughout this article as a shorthand for this pattern of posterior temporoparietal damage. Overall lesion size did not correlate with patients' background semantic scores $(r=-.35$ to .37 , $p>$.46) or performance on the semantic control tasks described below $(r=-.58$ to $.38, p>.2)$.

\section{Controls}

Two groups of eight control participants took part in this study. The second group participated in Experiment 3 only. None of the controls had a history of psychiatric or neurological disorder. Control groups did not differ from the patients, or each other, in terms of age $(t<1.5, p>$ .1) or educational level $(t<1.3, p>.2)$. 


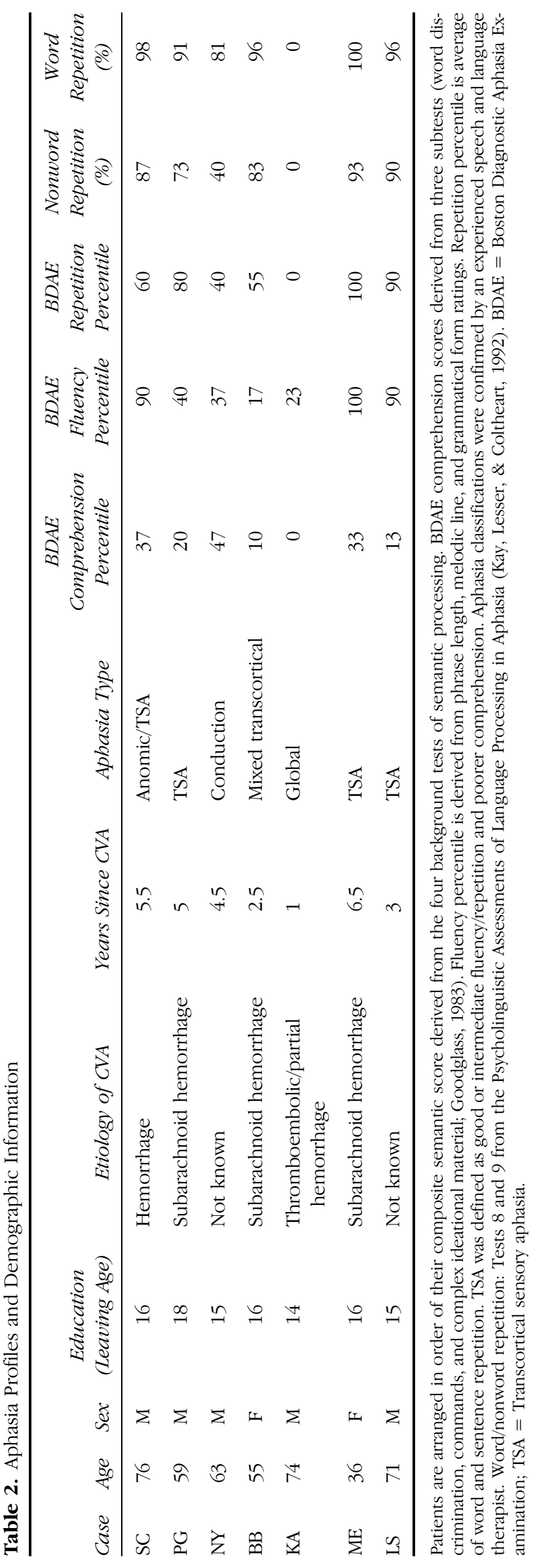

\section{BACKGROUND NEUROPSYCHOLOGICAL ASSESSMENT}

Method

General Neuropsychological Tests

A range of tests were used to assess executive/attentional functions: (a) the Ravens Coloured Progressive Matrices test of nonverbal reasoning (Raven, 1962), (b) the Wisconsin Card Sorting test (Stuss et al., 2000; Milner, 1964), (c) Elevator Counting with and without distraction from the Test of Everyday Attention (Robertson, Ward, Ridgeway, \& NimmoSmith, 1994), (d) the Brixton Spatial Rule Attainment task (Burgess \& Shallice, 1997), and (e) the Hayling Sentence Completion test (Burgess \& Shallice, 1997), which contrasts the production of appropriate sentence endings (e.g., "It is hard to admit when one is... wrong") with nonsensical endings that require the prepotent word to be suppressed (e.g., "Most sharks attack very close to... cups"). Visualspatial processing was assessed using the Visual Object and Space Processing (VOSP) battery (Warrington \& James, 1991), and working memory was examined using forward and backward digit span (Wechsler, 1987).

\section{Background Semantic Memory Assessments}

The presence of multimodal semantic impairment was assessed using a battery of semantic tests that tapped different input and output modalities for the same 64 items (Bozeat et al., 2000). There were four test components:

1. Spoken word-picture matching: The patients were required to match a verbally presented word to a target picture presented alongside nine semantically related foils. The pictures were black and white line drawings taken from the Snodgrass and Vanderwart (1980) corpus.

2. Picture naming: The patients named these drawings presented individually.

3. Camel and Cactus Test (CCT; Bozeat et al., 2000): This test of semantic associations is similar to the Pyramids and Palm Trees test (Howard \& Patterson, 1992). Patients were asked to decide which of four items was most associated to a probe item: for example, "camel" with "cactus," "rose," "tree," or "sunflower?" In two separate versions of this test, the same items were presented as pictures and as written words that were read aloud by the experimenter.

A composite score reflecting each patient's overall semantic abilities was derived from these four semantic tests using factor analysis. The patients are ordered by this composite score in the graphs and tables.

\section{Results}

The patients were impaired to varying degrees on all of the components of the semantic battery (see Table 1). Without exception, they also showed evidence of executive/ attentional impairment. Indeed, Jefferies and Lambon Ralph 


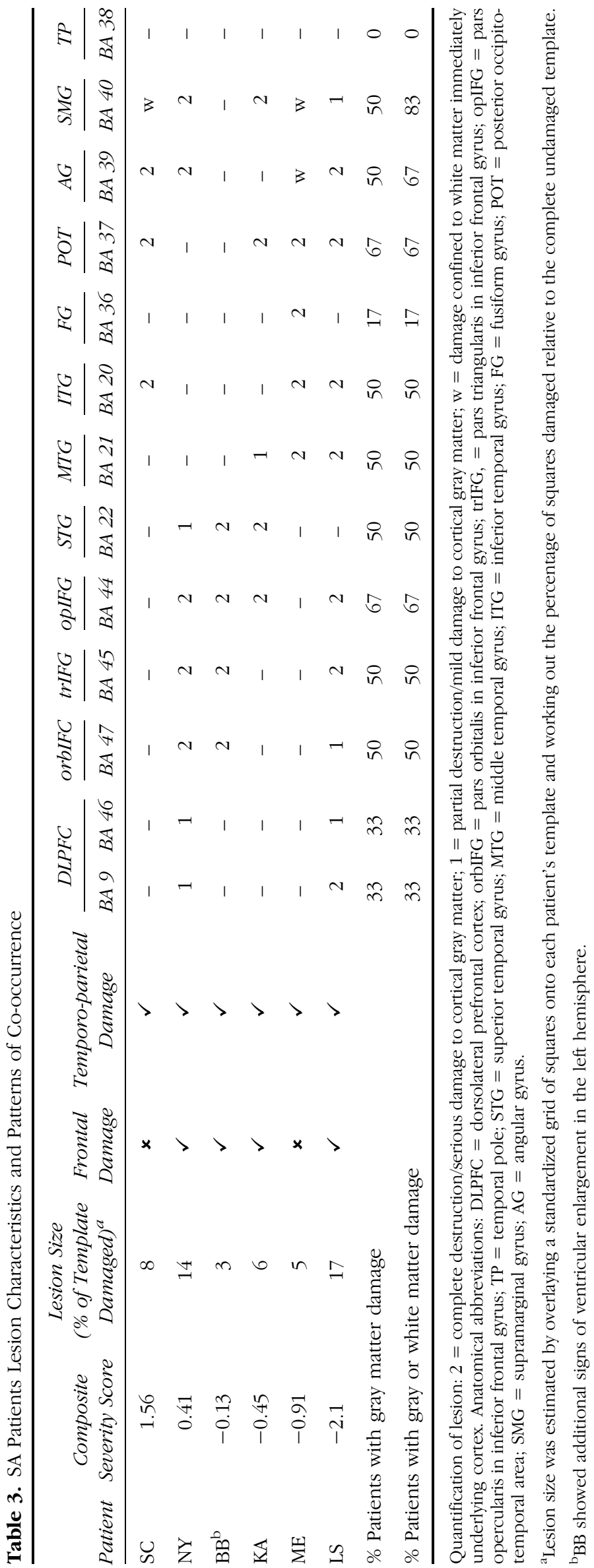


(2006) found that the severity of the semantic impairment in SA correlated with the degree of executive dysfunction. Some of the patients showed additional deficits in visualspatial processing and digit span.

\section{EXPERIMENT 1: DISTANCE THROUGH SEMANTIC SPACE}

The first experiment tested the patients' ability to manipulate and to explore semantic knowledge on-line. We used a semantic judgment task in which participants were required to indicate which of three alternatives was closest in meaning to a probe item. The probes and the targets were never identical in meaning; therefore, this task required multiple comparisons of semantic distances rather than identity matching per se (in contrast to synonym judgment where probes and targets have the same meaning). The degree of control required was manipulated by varying the semantic distance between probes and their targets while keeping the distractors the same. When probes and targets are close in semantic space (e.g., hat and cap), the items share a large amount of semantic structure and their similarity is relatively easy to discern. However, when the relationship is more distant (e.g., hat and stocking), additional semantic control may be required to work out the relevant semantic link. In addition, in this situation, it is harder to find the probe amongst the two distractors (e.g., futon, spade) as all three choices are a considerable distance from the probe in semantic space. Thus, it is harder to fathom which of these more distant relationships is the nearest. Therefore, SA patients with impaired semantic control should perform more poorly when the probe-target distance is greater.

\section{Method}

Participants were presented with a probe word and had to judge which of three accompanying words was closest in meaning. The words were presented as written stimuli and were also read aloud by the experimenter. Participants were instructed to respond as quickly and accurately as possible. Stimuli consisted of 64 concrete nouns drawn from eight semantic categories (animals, birds, plants, fruit/vegetables, tools, clothes, vehicles, and household objects) and two domains (natural and man-made things). The semantic distance between the probe and the target was manipulated to create two conditions. In half the trials (64/128), the probe and the target were distantly related while sharing membership to the same broad semantic category (e.g., chipmunk and bee = animals). In the remaining closely related trials, probe and target shared membership to a more specific subcategory in addition to their broader categorical similarities (e.g., chipmunk and squirrel $=$ rodents/animals). Target words in the distant condition also served as closely related targets on other trials (e.g., wasp and bee), allowing the same words to be presented in the two conditions. Distractor items were drawn from different semantic categories than the probe/target (e.g., chipmunk presented with wheat and cherry). Testing was completed over two sessions such that the close and distant versions of items did not occur within the same session. Table 4 provides further examples of the test stimuli.

\section{Results}

The results are shown in Figure 1. A two-way ANOVA revealed main effects of group, $F(1,13)=83.9, p<.001$, and semantic distance, $F(1,13)=47.4, p<.001$. As predicted by the semantic control hypothesis, a significant interaction was detected, $F(1,13)=34.8, p<.001$. SA patients performed significantly more poorly on distant judgments, $t(6)=6.1, p=.001$, planned comparison. Control subjects showed a trend in the same direction which did not reach significance, $t(7)=2.3, p=.054$. Individually, six of the seven SA patients showed significantly better performance for semantically close targets $\left(\chi^{2}=7.2\right.$ to 25.5 , one-tailed $p<.005$ ). Both posterior patients (ME

Table 4. Example Stimuli From the Semantic Distance Task

\begin{tabular}{|c|c|c|c|c|c|c|}
\hline \multirow[b]{2}{*}{ Stimulus } & \multicolumn{2}{|c|}{ Close } & \multicolumn{2}{|c|}{ Distant } & \multicolumn{2}{|c|}{ Distracters } \\
\hline & Target & Relationship & Target & Relationship & 1 & 2 \\
\hline Broccoli & Cauliflower & Vegetables & Apple & Plants & Lobster & Ostrich \\
\hline Buzzard & Eagle & Birds of prey & Gull & Birds & Ivy & Bean \\
\hline Elm & Oak & Trees & Wheat & Plants & Lion & Swan \\
\hline Hat & Cap & Headwear & Stocking & Clothes & Futon & Spade \\
\hline Leopard & Lion & Cats & Octopus & Animals & Rose & Coconut \\
\hline Ship & Yacht & Boats & Van & Vehicles & Radio & Hammer \\
\hline Shower & Bath & Bathroom objects & Oven & Household objects & Canoe & Shoe \\
\hline Watering can & Hosepipe & Garden tools & Spanner & Tools & Yacht & Belt \\
\hline
\end{tabular}


Figure 1. Impact of semantic distance in SA. Error bars show SEM.

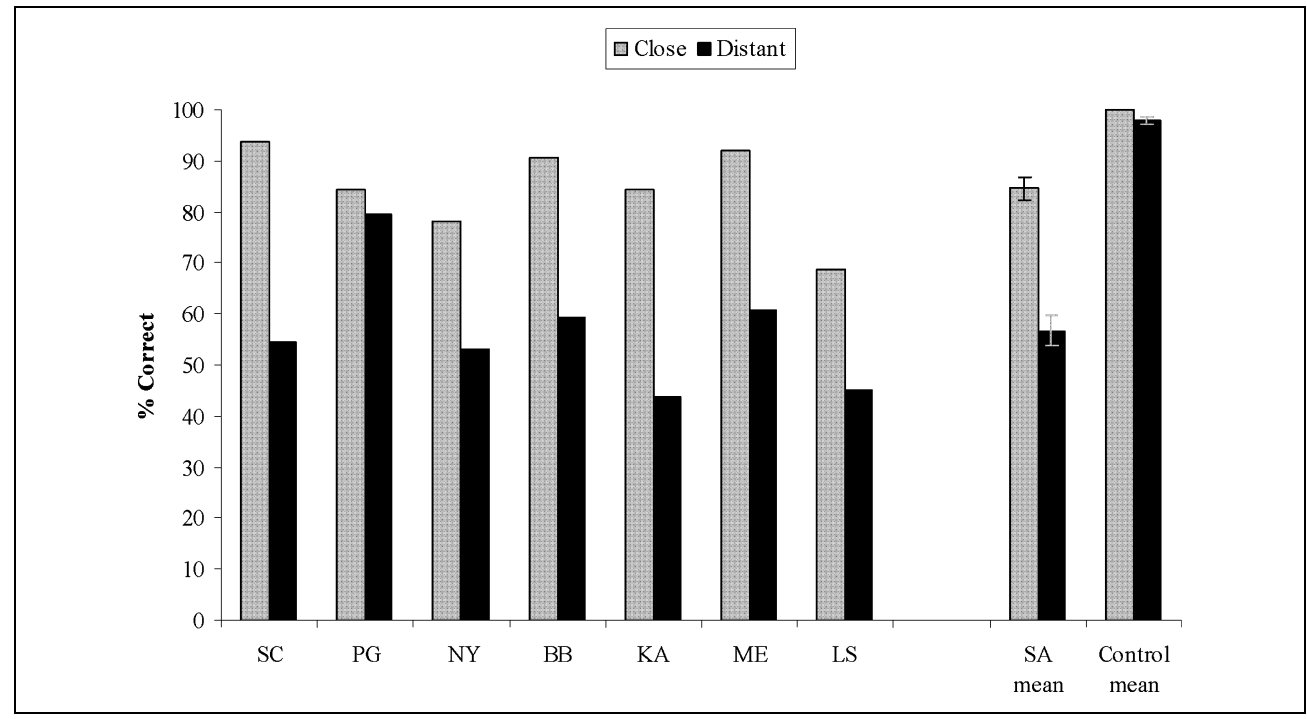

and SC) showed the same profile as those who had prefrontal involvement. PG showed no difference between conditions $\left(\chi^{2}<1\right)$.

\section{EXPERIMENT 2: SYNONYM/ANTONYM JUDGEMENT WITH HIGHLY ASSOCIATED DISTRACTORS}

The second experiment explored the patients' ability to inhibit irrelevant distractors in judgments of word meaning. As noted above, when two concepts are strongly related, then their relationship becomes hard to ignore even when they pertain to a task-irrelevant dimension (Samson et al., 2007; Badre et al., 2005; Wagner et al., 2001). This experiment examined the degree to which SA patients are disrupted by the use of highly associated distractors. The tasks (taken from Samson et al., 2007) examined synonym and antonym judgment when the associative strength between the probe and one of the distractors was either stronger or weaker than the association between the probe and the target. SA patients should have difficulty selecting the target on trials containing strongly associated distractors because executive control is required to overcome competition (and perhaps boost the target) in this context.

\section{Method}

\section{Synonym/Antonym Judgment With Highly Associated Distractors}

The design exactly replicated Experiment 1 from Samson et al. (2007). Participants were presented with a probe word and were asked to judge which of three accompanying words had either the same meaning (in one block of 144 trials) or the opposite meaning (in a second block of 144 trials). The words were presented as written stimuli and were also read aloud by the experimenter. Participants were instructed to respond as quickly and accurately as possible. The array of choices was composed of a synonym, an antonym, and an unrelated foil in every trial. Moreover, the same words were used in the synonym/antonym tasks (e.g., happy with cheerful, sad, or conscious) but their use as targets/distractors changed (see Figure 2A). The associative strength of the distractor antonym/synonym word was manipulated so that it was either strongly or weakly associated with the probe word according to word association norms (for further details, see Samson et al., 2007). This resulted in a total of four conditions, each composed of 72 items: (a) synonym judgment with weak distractors; (b) synonym judgment with strong distractors; (c) antonym judgment with weak distractors; and (d) antonym judgment with strong distractors (see Figure $2 \mathrm{~A}$ ). The

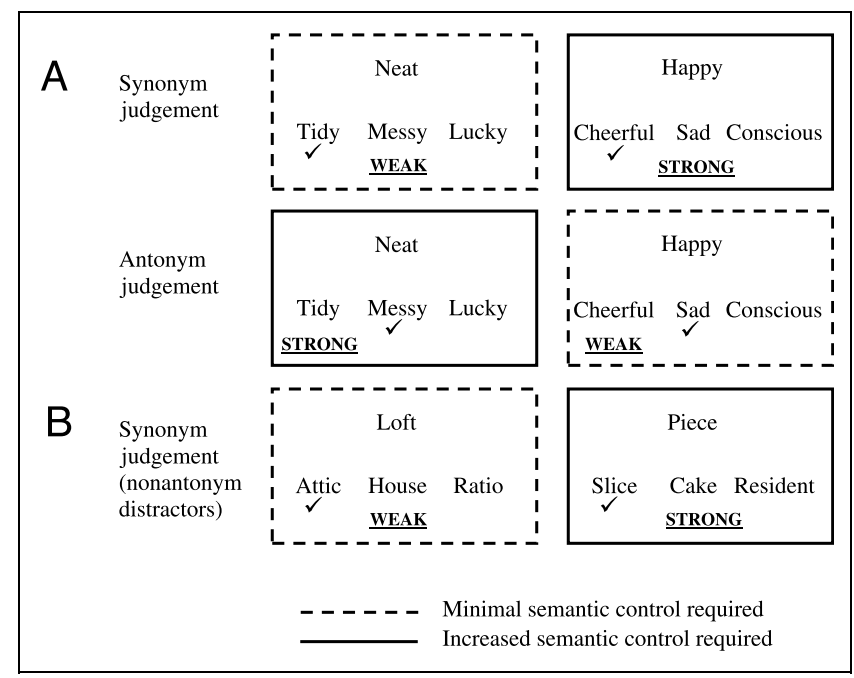

Figure 2. Structure of synonym/antonym task (from Samson et al., 2007). Dashed borders represent conditions in which semantic control demands are minimised (i.e., the distractor foils are weakly associated to the probes). Unbroken borders represent conditions with increased semantic control demands (i.e., the distractor foils are strongly to the associated probes). Ticks/check marks denote the correct response for each example trial. 
stimuli included nouns, verbs, adjectives, adverbs, and prepositions, but within any given trial, all of the words were drawn from the same grammatical class. The synonym and the antonym tasks were presented in separate sessions at least 2 weeks apart. To explain the task requirement to the patients, the tasks were introduced using easy practice items accompanied by pictures (e.g., woman with man and lady). Within each testing block, the strong and the weak distractor trials were mixed together.

\section{Synonym Judgment With Associated (Nonantonymous) Distractors}

A second version of the test was also administered, which exactly replicated Experiment 2 from Samson et al. (2007). In this test, distractors shared a semantic association with the probe word without being a synonym/antonym. For example, the probe "piece" was presented with the target "slice" and accompanied by "cake" as an associated distractor and "resident" as an unrelated distractor (see Figure 2B). This test consisted of 88 items, which were presented to the patients in a single block. Again, the strong and the weak distractor trials were mixed together.

\section{Results}

The results are shown in Figure 3. A three-way ANOVA detected main effects of distracter type, $F(1,13)=9.5, p<$ .01 , and group, $F(1,13)=42.6, p<.001$, but no influence of judgment type (antonym vs. synonym), $F(1,13)=1.5$, $p>$.2. The only significant interaction was between group and distracter type, $F(1,13)=7.5, p<.02$, reflecting the finding that SA patients were less accurate on judgments accompanied by strongly associated distracters, $t(6)=2.7, p<.05$, planned comparison, whereas controls were not, $t(7)=1.5, p=.15$. On the second task that examined synonym judgment with nonantonymous distracters, a two-way ANOVA revealed main effects of distracter type, $F(1,13)=20.9, p=.001$, and group, $F(1,13)=82.8$, $p<.001$. Moreover, there was a significant group by distracter type interaction, $F(1,13)=9.7, p=.01$, indicating that the disadvantage for strongly associated distracters was greater in SA patients, $t(6)=3.7, p<.01$, planned comparison, than controls, $t(7)=2.3, p<.05$.

At the level of individual patients, five cases showed significantly better performance on trials with weakly associated distracters, regardless of whether they had damage to $\mathrm{pFC}$ or not (SC, PG, BB, KA, and ME; $\chi^{2}>2.8$, one-tailed $p<.05$ to $p<.001$, combining across the three test sessions). LS and NY showed no difference between conditions $\left(\chi^{2}<1.4, p>.1\right)$, although LS's performance was close to floor.

\section{Summary}

As expected, the SA patients performed more poorly on synonym/antonym judgment when the distractor words were highly associated with the probe. These findings are consistent with the view that poor executive control prevents SA patients from overcoming interference from activated but irrelevant concepts in semantic tasks.

\section{EXPERIMENT 3: EFFECT OF CUES AND MISCUES ON PICTURE NAMING}

Previous studies have shown that SA patients are far more responsive than SD patients to phonemic cues in picture naming tasks (Jefferies et al., 2008). Phonemic cues boost activation of target words relative to semantically related competitors and, consequently, circumvent SA patients' difficulties in regulating semantic activation. For example, if the prepotent associate "tea" is activated in response to a picture of a cup, this error will be inconsistent with the phonemic cue $/ \mathrm{k} /$, increasing the likelihood of a correct response. These findings also show that SA patients retain knowledge of picture names that they cannot retrieve unaided. Cueing might be less beneficial in SD because these patients do not have difficulties directing their residual semantic activation appropriately and/or because there is less semantic activation with which a cue can combine.

If SA patients do suffer from deregulated semantic cognition, then it should be possible to manipulate their performance in both a positive and a negative manner. To test this possibility, we examined the impact of phonemic miscues on picture naming (Lambon Ralph, Sage, \& Roberts, 2000; Howard \& Orchard Lisle, 1984). The patients were miscued with the initial phoneme of a word that was semantically related to the target (e.g., picture of knife + /f/ from fork). The miscues were designed to strengthen the activation of a close semantic competitor, thereby impairing picture naming in SA.

\section{Method}

This test was taken from Lambon Ralph et al. (2000). A total of 140 black and white line drawings were tested in three conditions: (a) uncued picture naming, (b) picture naming with a correct cue that was the first phoneme of the target word, and (c) miscued naming, in which pictures were presented alongside the initial phoneme of a semantically related item (e.g., knife $+/$ f/ from fork). To aid their articulation, the single consonant cues/miscues were combined with a schwa. In each session, one third of the items were assigned to each condition. Conditions were changed in a Latin-square fashion across three testing sessions such that, at the end, all items had appeared in all three conditions. Different types of cue were interleaved across the items within a session. The participants were told that the initial phoneme cue might be helpful on some trials but not others. Participants were instructed to name the pictures as quickly and accurately as possible. 
Figure 3. Impact of distractor associative strength on synonym (A) and antonym (B) judgment and on synonym judgment with nonantonymous distractors (C).
A Synonym judgment

- Highly associated distractor $\square$ Weakly associated distractor

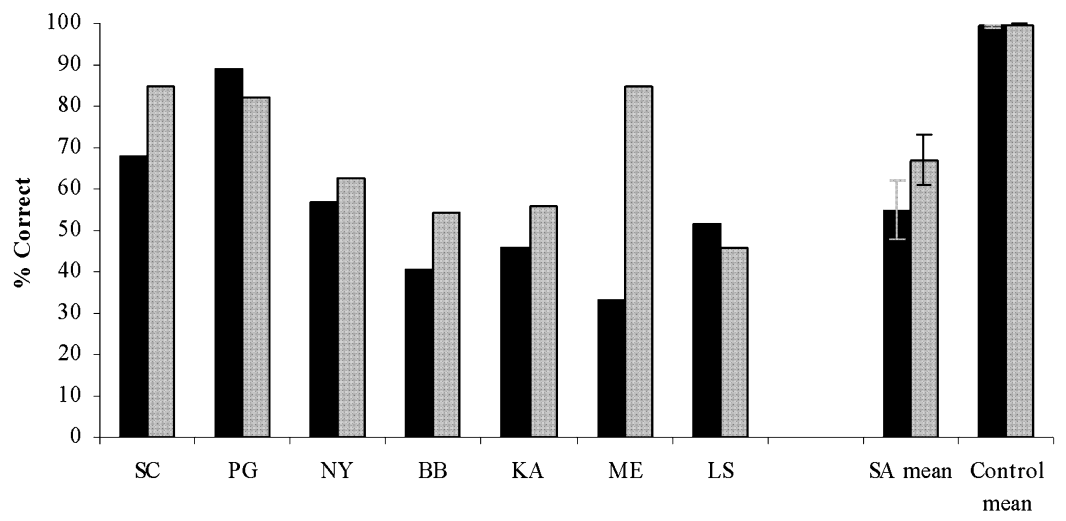

B Antonym judgment

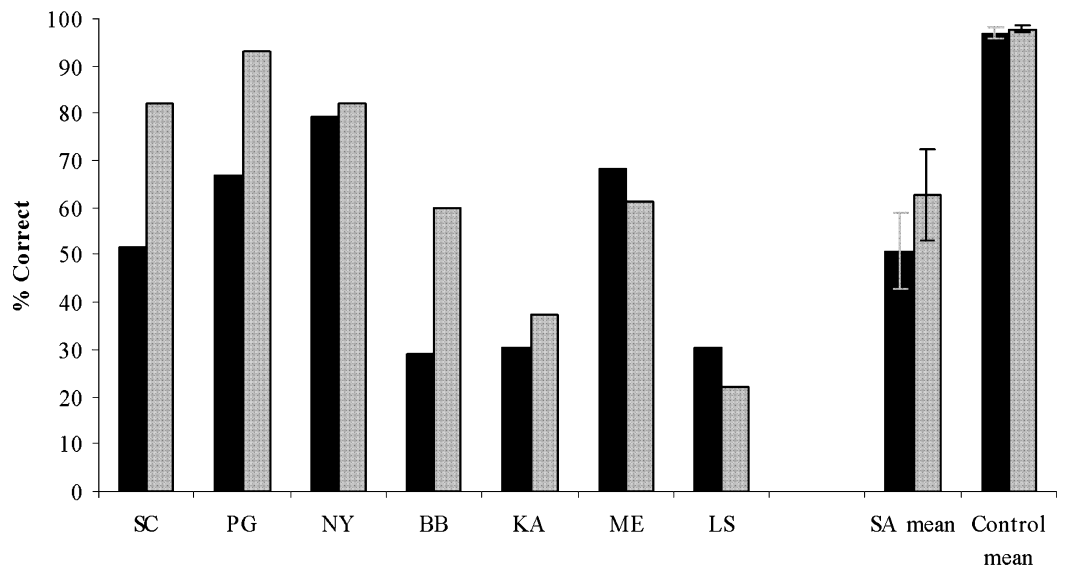

C Synonyms with nonantonymous distractors

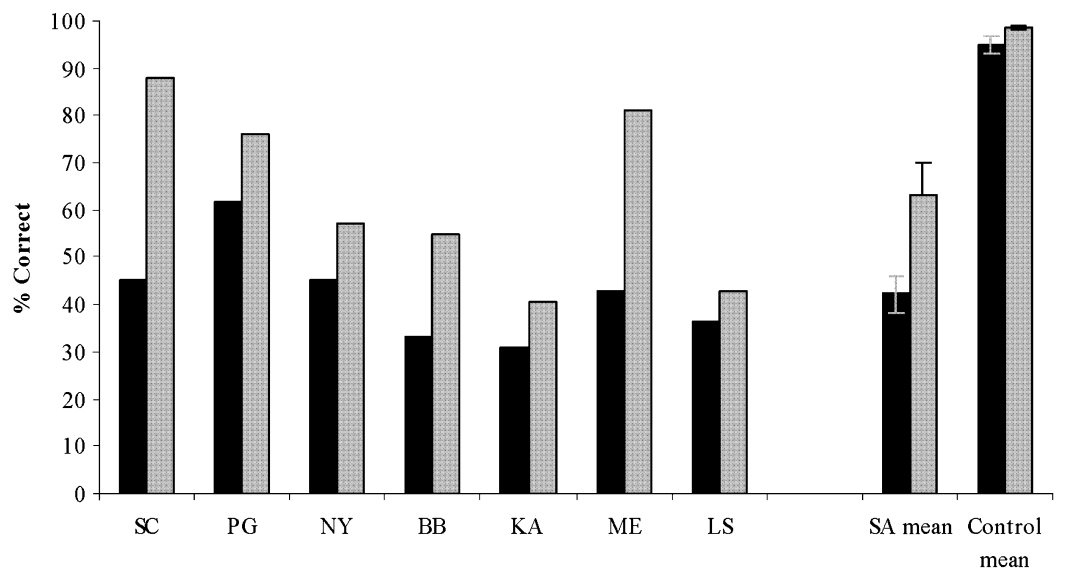

Error bars show SEM
Patient KA, who had very poor spoken output, was not tested on this task.

\section{Results}

Picture naming accuracy without cues and following phonemic cues/miscues is shown in Figure 4. These data exclude self-corrections (allowing miscued responses that were rapidly corrected to be counted as errors). A two-way ANOVA revealed main effects of cueing, $F(2$, $24)=12.7, p<.001$, and group, $F(1,12)=47.9, p<$ .001 , as well as a significant interaction between group and cueing, $F(2,24)=11.2, p<.001$. Relative to baseline, SA patients showed both enhanced naming following 
Figure 4. Effects of phonemic cueing and miscuing on accuracy of picture naming. Error bars show SEM.

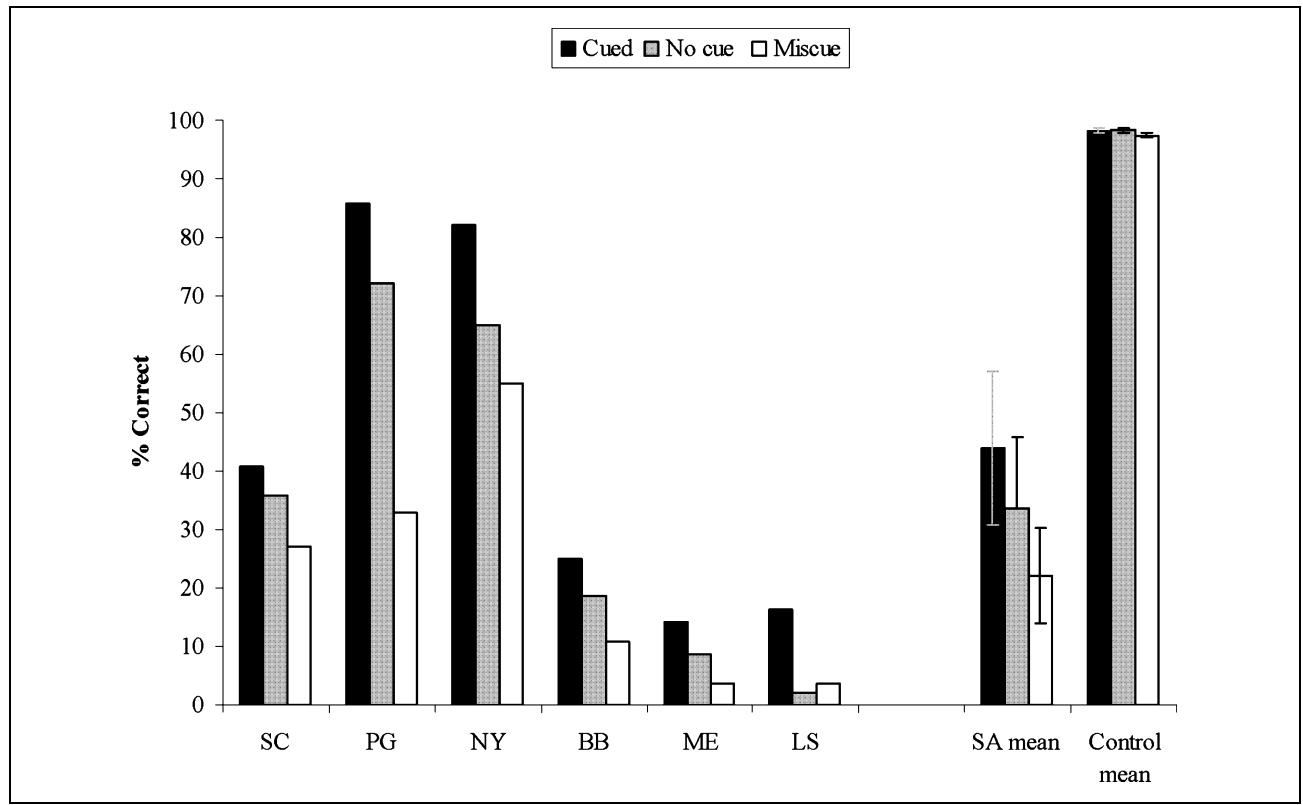

phonemic cues, $t(5)=4.8$, one-tailed $p=.002$, planned comparison, and decreased naming following miscues, $t(5)=1.9, p=.05$. Controls did not show an advantage for cued relative to uncued naming, $t(7)<1$, although they did show a miscuing effect, $t(7)=2.2, p=.03$.

Every SA patient showed more successful picture naming performance with appropriate cues than miscues, regardless of whether they had damage to $\mathrm{pFC}$ or not (McNemar one-tailed exact $p=.01$ to <.0001). Correct cues improved naming accuracy relative to baseline for NY, PG, ME, and LS (McNemar one-tailed exact $p=.04$ to <.0001) and the cueing effect approached significance for $\mathrm{BB}(p=.09)$. SC did not show a significant cueing effect $(p=.18)$, although he showed an effect with longer phonemic cues in a previous study (Jefferies et al., 2008). In addition, the majority of the SA group showed poorer naming following miscues relative to baseline. This difference was significant or nearly so for $5 / 6$ patients (McNemar one-tailed exact $p=.06$ to <.0001). The effect of miscues could not be assessed for the final patient, LS, as his baseline naming was at floor.

The errors made by individual patients are shown in Figure 5. The number of semantic errors, expressed as a proportion of total errors, differed across the uncued, cued, and miscued conditions (Friedman test), $\chi^{2}(2)=$ $9.3, p=.01$. Miscued trials generated more semantic errors than both correctly cued and uncued trials (Wilcoxon signed ranks, $Z=2.2, p=.03$ for both comparisons). In the miscued condition, there were frequent semantic errors that were phonologically consistent with the cue (e.g., picture of a table $+/ \mathrm{ch} / \rightarrow$ "chair"). Therefore, the SA patients showed a miscuing effect in errors as well as accuracy. Every SA patient made more cue-consistent semantic errors as a proportion of total errors following mis- cues compared with correct cues (Fisher's exact one-tailed $p=.0004$ to .08$)$.

\section{Summary}

This experiment explored the effect of phonemic cues and miscues on picture naming in SA. The miscues, which were designed to increase the degree of competition with the target response, impaired the SA patients' ability to name the pictures correctly and elicited additional semantic errors. These findings confirm that the SA patients had difficulty directing activation toward appropriate targets and away from semantic competitors.

\section{EXPERIMENT 4: SEMANTIC AMBIGUITY AND THE INFLUENCE OF CUEING}

In the final experiment, we tested the patients' ability to boost less intrinsically dominant meanings when required for a specific task (another standard feature of control mechanisms). We achieved this by testing comprehension of polysemous words. Previous research suggests that when ambiguous items are encountered, their alternative meanings are activated in parallel (Rodd, Gaskell, \& MarslenWilson, 2004; Simpson \& Burgess, 1984; Onifer \& Swinney, 1981). The degree of competition between these alternative interpretations is determined, at least in part, by the relative frequency of each meaning: less frequent meanings show a processing disadvantage (Simpson, 1985). In healthy individuals, semantic control processes are thought to play a role in selecting the less common meaning of homonyms when appropriate and avoiding the dominant but incorrect interpretation. Recent neuroimaging studies 
Figure 5. Effects of phonemic cues and miscues on picture naming errors.

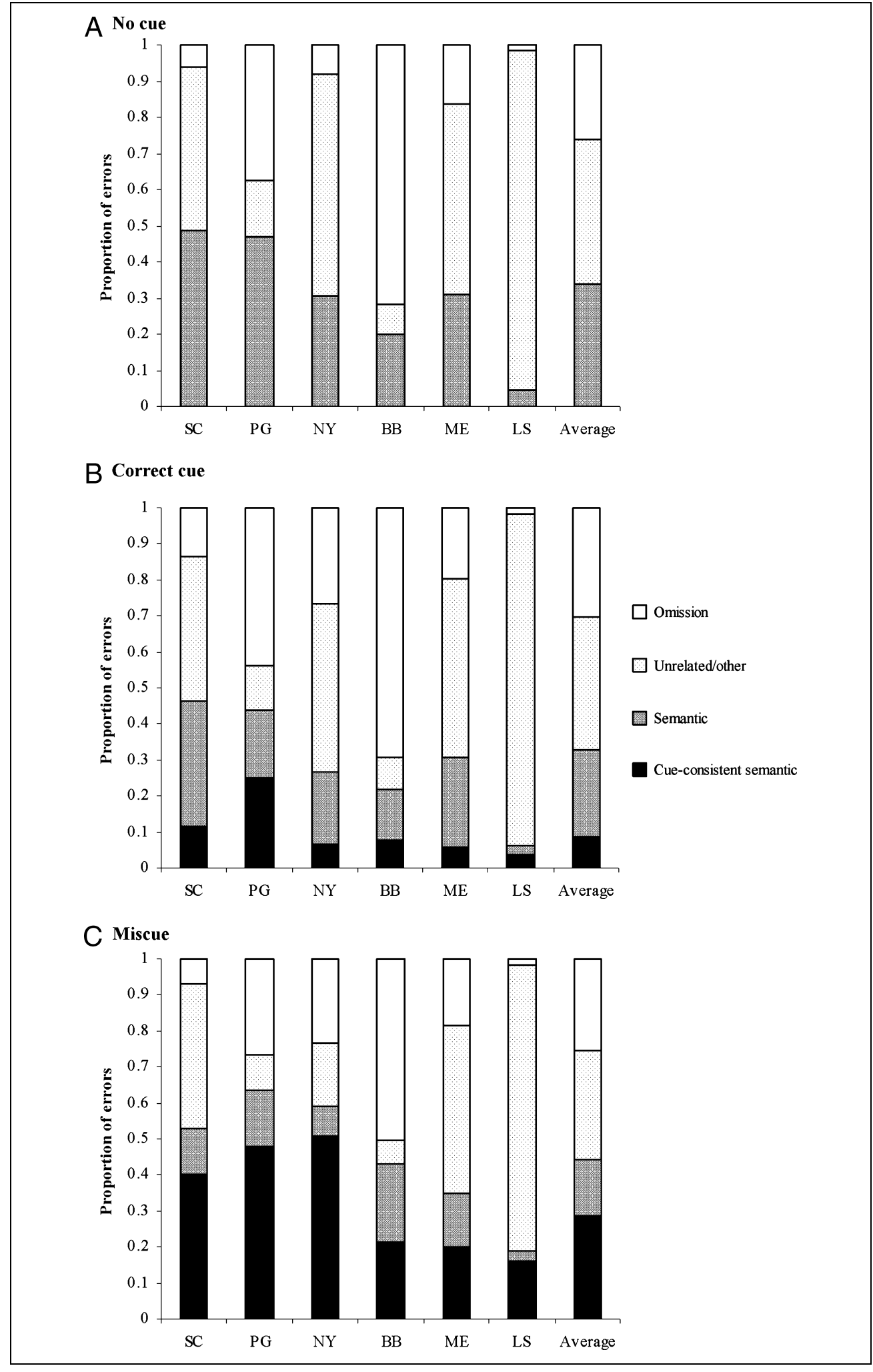

have shown that resolving semantic ambiguity is associated with activation in the left inferior frontal gyrus and posterior temporal cortex (Zempleni et al., 2007; Rodd et al., 2005), areas which are damaged in SA patients with impaired semantic control.
This experiment examined the ability of SA patients to process dominant and less frequent meanings of homonyms in a semantic judgment task. We predicted that SA patients would struggle to select the less frequent interpretations of ambiguous words, reflecting their poor 
control over semantic processing. We also tested our working hypothesis that in SA, it is not the semantic representations themselves that are damaged but rather the control processes that manipulate or shape the aspect of meaning that is relevant to the current task. We achieved this by examining the effect of sentence contexts, which either cued the relevant meaning or miscued the opposing meaning of the homonyms. If SA cases have poor semantic control but intact representations of the various meanings of ambiguous words, correct cues should allow these patients to retrieve the less frequent meanings of homonyms. Moreover, miscues should impair the performance of SA patients by increasing the activation of competing meanings.

\section{Method}

Participants were asked to select which of four words was related in meaning to a probe word presented at the top of the page. The target was presented alongside two unrelated foils. All five words were presented in a written format and were also read aloud by the experimenter. In half of the trials, the target referred to the dominant meaning of the probe word (fire $=$ hot). The remaining trials required the participants to make a link between the target and the subordinate meaning of the probe (fire $=$ rifle). Thirty ambiguous probe words were selected using the free association norms of Twilley, Dixon, Taylor, and Clark (1994). These norms provide a measure of the relative frequency of the alternative meanings (i.e., the proportion of subjects who generated words linked to each meaning in the free association task). We selected stimuli that showed a large difference in frequency between the two alternative meanings, such that one interpretation of the word was dominant over the other (e.g., fire $=$ hot selected by $87 \%$ of subjects; fire $=$ rifle selected by 3\%). Target words for the dominant and the less common meanings were matched for lexical frequency, $t(58)<1$, and imageability, $t(58)<1$. The distractor foils on a given trial were matched to the average lexical frequency/imageability of the two target words. In addition, the same distracters were used on the trials that tested the two alternative meanings of the probe word.

Performance on these materials was examined under three cueing conditions: no cues, correct cues, and miscues. The cue took the form of a sentence which either primed the appropriate semantic meaning (e.g., fire on a dominant trial: "I lit a fire") or the opposing meaning (e.g., "Fire at will"). The sentences were presented in a written format and simultaneously read aloud to the participants immediately before each trial to be cued or miscued. Therefore, the semantic judgment task was interleaved with sentence reading/listening but the task instructions remained the same: The participants were asked to select the word related in meaning to the probe word as quickly and accurately as possible. They were told that the sentences might be helpful on some trials but not others.

Testing was carried out over a total of four sessions, each separated by a minimum of 2 weeks. The uncued condition was presented first over two sessions. The alternative meanings of the same words were not tested within the same session. Cued and miscued trials were tested in a further two sessions. Within these sessions, both meanings of each item were probed (e.g., fire = hot vs. fire $=$ rifle), whereas the sentence context remained constant (e.g., "I lit a fire").

\section{Results}

The results are shown in Figure 6. A three-way ANOVA revealed main effects of group, $F(1,6)=74.3, p<.001$, dominance, $F(1,13)=11.58, p<.005$, and cueing, $F(1$, $13)=35.88, p<.001$. SA patients were more strongly affected than controls by both experimental manipulations resulting in significant group by task interactions, dominance, $F(1,13)=6.16, p<.05$; cueing, $F(2,26)=29.51$, $p<.001$. Moreover, there was a three-way interaction, indicating that cueing had a greater influence on SA patients' ability to access the less common meanings of items, $F(1,13)=8.0, p<.005$. In the uncued and the miscued conditions, SA patients' comprehension was poorer for the less frequent meanings, $t(6)=2.87, p<.05$ and $t(6)=$ $3.19, p<.05$. However, the provision of cues substantially improved performance for the less frequent meanings such that the difference between the dominant and the less common meanings was eliminated, $t(6)<1$. In contrast, control participants only showed a disadvantage for the infrequent meanings in the miscued condition, $t(7)=$ 2.36 , uncorrected $p=.05$. There was no difference in the uncued, $t(7)=1.9, p=1$, or cued, $t(7)=1.5, p>1$, conditions.

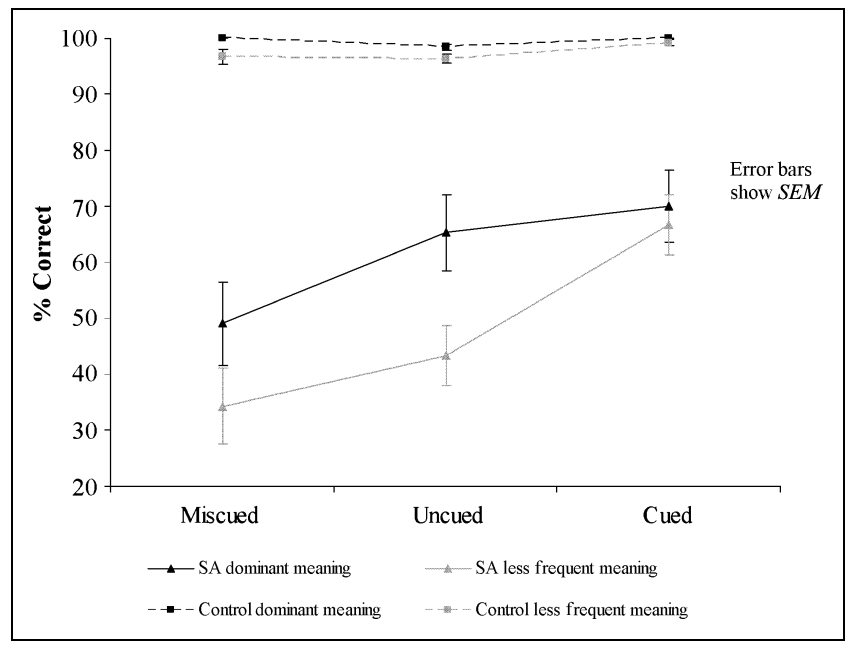

Figure 6. Influence of cueing context on access to dominant and less frequent meanings of homonyms in SA. 
Table 5. Influence of Cueing Context on Access to Dominant and Less Frequent Meanings of Homonyms

\begin{tabular}{lccccccc}
\hline & $S C$ & $P G$ & $N Y$ & $B B$ & $K A$ & $M E$ & $L S$ \\
\hline Dominant & & & & & & & \\
Miscued & 76 & 46 & 60 & 40 & 40 & 63 & 16 \\
Uncued & 86 & 63 & 76 & 46 & 70 & 76 & 36 \\
Cued & 83 & 90 & 73 & 56 & 66 & 80 & 40 \\
& & & & & & & \\
Less Frequent & & & & & & \\
Miscued & 73 & 33 & 33 & 26 & 20 & 30 & 23 \\
Uncued & 66 & 56 & 43 & 43 & 26 & 33 & 33 \\
Cued & 80 & 86 & 73 & 63 & 60 & 56 & 46 \\
\hline
\end{tabular}

Scores represent percentage correct.

Individual patient data are given in Table 5. Four patients showed better performance on trials involving dominant as opposed to infrequent meanings (SC, NY, KA, and ME; $\chi^{2}>3.35$, one-tailed $p=.03$ to $p<.001$, data from the uncued condition). In addition, six of the seven patients were more accurate in the cued than the miscued trials (McNemar one-tailed exact $p=.007$ to $<.001$, combining the dominant and less frequent meanings). SC did not show any cueing effects $(p>.2)$. ME, one of the patients with posterior only damage, showed an identical profile to the patients with prefrontal involvement.

\section{Summary}

As expected, SA patients had difficulty retrieving the less frequent meanings of ambiguous words and showed significant cueing and miscuing effects in this comprehension task. Moreover, we observed an interaction between dominance and cueing: The provision of a sentence cue particularly benefited the retrieval of less frequent meanings in SA, bringing the accuracy of these items up to the level seen with dominant meanings. These findings support the view that SA patients have poor control over semantic activation but a broadly intact representation of both the dominant and the less frequent meanings of ambiguous words.

\section{GENERAL DISCUSSION}

This study examined the hypothesis that multimodal semantic impairment in stroke aphasia (referred to here as SA) follows from a deficit of executive control over activation within the semantic system, as opposed to a loss of semantic knowledge per se. By systematically varying the nature and the degree of control required in the tasks used, this study was able to reveal the nature and quality of semantic control deficits for the first time.
The view that semantic cognition-semantically driven behavior-is underpinned by two interactive principal components (semantic representation and control) is motivated by a recent study that observed qualitatively different patterns of semantic impairment in patients with SA and SD (Jefferies \& Lambon Ralph, 2006). Although both groups of patients had the same degree of difficulty on semantic tasks involving different input and output modalities (auditory/written words, pictures, environmental sounds; matching, speech), they showed clear differences in (a) the degree of consistency across semantic tasks that had different control requirements, (b) the effect of item frequency/familiarity, (c) the extent to which performance was predicted by controls' ratings of the executive difficulty of each trial, (d) the nature of their picture naming errors, and (e) the degree to which interference built up in cyclical naming and word-picture matching. Jefferies and Lambon Ralph (2006) suggested that this divergence was best explained as a disruption of semantic control processes in SA, as opposed to a degradation of semantic representations in SD. However, this earlier study did not include any tasks that directly varied the requirement for semantic control. The current investigation addresses this gap, demonstrating for the first time that SA patients are highly sensitive to manipulations of the executive demands of semantic tasks. We used several different methods to produce high- and low-semantic control conditions across four experiments and to manipulate the different types of control required. These can be summarized as follows:

1. Impaired on-line manipulation and exploration of semantic knowledge: The SA patients had greater difficulty making connections between items that were further apart in semantic space (e.g., chipmunk with bee) than between more similar items (chipmunk with squirrel). They struggled to discern the relevant relationship when there was less semantic overlap between the target and the probe. This finding is consistent with the view that poor semantic control in SA impairs the patients' on-line manipulation and exploration of semantic knowledge. Similarly, Jefferies and Lambon Ralph (2006) found that SA patients were more sensitive than SD patients to ambiguity surrounding the relevant relationship when making judgments of semantic association (as measured by controls' ratings).

2. Poor inhibition of strongly associated distractors: In synonym/antonym judgment, the rate of errors increased when the distractors were highly associated with the probe items, suggesting that the SA patients had difficulty directing activation away from irrelevant but prepotent associates.

3. Poor on-line control of target versus distractor activation: The SA patients' picture naming showed positive effects of phonemic cues: they were able to name significantly more items when provided with the initial phonemes of the targets. The phonemic cues would have ruled out potential competitors at the same time as 
boosting activation of the correct response. Moreover, the SA patients showed negative effects of miscues (i.e., the first phoneme of a semantically related word, e.g., "ch" for table). The miscues were designed to increase activation of a semantic competitor and led to a greater number of semantic errors. These findings confirm that the SA patients had difficulty focussing activation on the target and away from competing responses, consistent with the hypothesis of poor semantic control.

4. SA reflects poor semantic control not impaired semantic representation: The patients' primary deficit did not appear to be a loss of semantic information per se because they could demonstrate knowledge in one context that they were unable to express in another. This effect was observed both in picture naming and in the final comprehension task. The SA patients had difficulty retrieving the less common meanings of ambiguous words when making semantic judgments (e.g., bank = money better than bank $=$ river $)$. They were able, however, to respond accurately when the relevant interpretations of the ambiguous words were cued. Following cueing, the patients showed equivalent performance for dominant and subordinate meanings, indicating that the less frequent meanings were not lost from the semantic system. In addition, they were readily miscued when provided with a context that activated the irrelevant meaning of the homonyms. Therefore, this experiment provides further evidence for a control problem in SA.

Consistent with the deficient semantic control hypothesis, the SA patients showed strong effects of executive demands on all four semantic tasks. By providing a better characterization of the nature of the semantic deficit in SA, the results help to explain why multimodal semantic impairment is associated with very different areas of brain damage in SA and SD. As outlined in the Introduction, we propose that semantic cognition is composed of two principal components: ATL conceptual representations and pFC-TPJ semantic control processes (Jefferies \& Lambon Ralph, 2006). Bilateral atrophy of the ATLs in SD produces a progressive degradation of semantic knowledge (Lambon Ralph \& Patterson, 2008; Patterson et al., 2007; Rogers et al., 2004). Although individuals with SA fail the same range of verbal and nonverbal semantic tasks as SD patients, their ability to retrieve semantic information clearly varies with the executive demands of the test/trial. This indicates that the store of semantic knowledge is relatively intact in SA. Instead, the patients have difficulty regulating and shaping activation on-line within the semantic system such that they fail to promote relevant aspects of knowledge, especially when it is intrinsically weak, and struggle to inhibit irrelevant elements of concepts. This explains why SA patients have difficulty in the face of strongly associated distractors in synonym judgment, why they struggle to discern the relevant semantic relationships when probed for both the less frequent meanings of ambiguous words and relationships that span greater dis- tances in semantic space, and why they show a vulnerability to miscues as well as a benefit of cues.

Poor semantic control in SA is associated with lesions of pFC and TPJ (Jefferies \& Lambon Ralph, 2006). Our findings are consistent, therefore, with functional neuroimaging studies of healthy subjects, which show greater activation within these regions in conditions requiring the controlled use of semantic knowledge. Although the functional neuroimaging literature has highlighted the role of the left inferior prefrontal gyrus in semantic control, activations within posterior temporal cortex (i.e., BA 37, 21) often show a similar profile (Badre et al., 2005; Wagner et al., 2001; Thompson-Schill et al., 1997). Furthermore, posterior temporal areas frequently show activationalong within the inferior frontal gyrus-when tasks require less dominant aspects of semantic knowledge to be brought to the fore, that is, when accessing the less common interpretation of an ambiguous word or the nonliteral meaning of a metaphor (Bedny et al., 2008; Gennari et al., 2007; Zempleni et al., 2007; Lee \& Dapretto, 2006; Rodd et al., 2005). Similarly, inferior parietal cortex (i.e., BA 39/ 40) has been implicated in the regulation of semantic knowledge. Orientating attention to semantic categories as well as switching between different clusters in semantic fluency produces activation in inferior parietal and inferior frontal cortices (Cristescu et al., 2006; Hirshorn \& Thompson-Schill, 2006). This suggests that regions in both inferior frontal and posterior temporal/inferior parietal cortex contribute to regulatory control in the semantic domain. It should be noted here-we are not implying that all temporo-parietal activations are solely related to semantic control. Specific areas within this region are activated when different semantic categories are contrasted and control demands are held constant (see Martin, 2008)implying that aspects of semantic representation and semantic control are underpinned by different parts of this broad region.

We propose, therefore, that semantic control is underpinned by processing within both $\mathrm{pFC}$ and TPJ. In the current study, SA patients with $\mathrm{pFC}$ and TPJ lesions showed identical effects of semantic distance, cueing/miscuing, and associative strength, consistent with earlier reports of parallel semantic deficits following damage to these two brain regions (Jefferies et al., 2007; Jefferies \& Lambon Ralph, 2006; Berthier, 2001). The notion that semantic control is dependent on interactions between $\mathrm{pFC}$ and TPJ was supported by neuroanatomical evidence indicating strong connectivity between these two areas via the arcuate and longitudinal fasciculi (Parker et al., 2005; Gloor, 1997). Furthermore, both pFC and parietal areas are recruited when healthy subjects perform tasks requiring attentional control, updating of working memory, and inhibitory processing (Rowe, Hughes, Eckstein, \& Owen, 2008; Collette, Hogge, Salmon, \& Van der Linden, 2006; Hon, Epstein, Owen, \& Duncan, 2006; Collette et al., 2005; Garavan, Ross, Li, \& Stein, 2000). pFC and TPJ lesions resembling those in our patients produce highly similar 
patterns of disruption to attentional processes beyond the semantic domain (Peers et al., 2005). This is consistent with the findings from our current study where patients showed evidence of nonverbal attentional/executive dysfunction on tasks not requiring conceptual knowledge. It seems likely, therefore, that semantic control relies on both anterior and posterior cortical areas, like other more general executive processes (Nagel et al., 2008). Further research will be required to ascertain if the semantic impairment in SA patients can be explained by a generalized problem with executive control.

\section{Acknowledgments}

The authors are indebted to the patients and their carers for their generous assistance with this study. They would like to thank Rachel Byrne, Linda Collier, and Claire Slinger for referring some of the patients to us. They gratefully acknowledge Karen Sage's help with classifying the stroke patients' aphasic syndromes. Additional thanks are offered to Dana Samson and Glyn Humphreys for the use of their synonym judgment task. The work was supported by a program grant from the MRC (G0501632), an MRC studentship to K. A. N., and an RCUK fellowship to E. J.

Reprint requests should be sent to Prof. Matthew A. Lambon Ralph, Neuroscience and Aphasia Research Unit (NARU), Zochonis Building, School of Psychological Sciences, University of Manchester, Oxford Road, Manchester, M13 9PL, UK, or via e-mail: matt.lambon-ralph@manchester.ac.uk, Web site: www.psych-sci.manchester.ac.uk/naru.

\section{REFERENCES}

Badre, D., Poldrack, R. A., Pare-Blagoev, E. J., Insler, R. Z., \& Wagner, A. D. (2005). Dissociable controlled retrieval and generalised selection mechanisms in ventrolateral prefrontal cortex. Neuron, 47, 907-918.

Badre, D., \& Wagner, A. D. (2002). Semantic retrieval, mnemonic control, and prefrontal cortex. Behavioral \& Cognitive Neuroscience Reviews, 1, 206-218.

Bedny, M., McGill, M., \& Thompson-Schill, S. L. (2008). Semantic adaptation and competition during word comprehension. Cerebral Cortex, 18, 2574-2585.

Berthier, M. L. (2001). Unexpected brain-language relationships in aphasia: Evidence from transcortical sensory aphasia associated with frontal lobe lesions. Aphasiology, 15, 99-130.

Borden, N. M. (2006). 3D angiographic atlas of neurovascular anatomy and pathology. Cambridge: Cambridge University Press.

Bozeat, S., Lambon Ralph, M. A., Patterson, K., Garrard, P., \& Hodges, J. R. (2000). Non-verbal semantic impairment in semantic dementia. Neuropsychologia, 38, 1207-1215.

Burgess, P. W., \& Shallice, T. (1997). The Hayling and Brixton tests. Bury St. Edmunds, UK: Thames Valley Test Company.

Caramazza, A., \& Shelton, J. R. (1998). Domain-specific knowledge systems in the brain: The animate-inanimate distinction. Journal of Cognitive Neuroscience, 10, 1-34.

Chertkow, H., Bub, D., Deaudon, C., \& Whitehead, V. (1997). On the status of object concepts in aphasia. Brain and Language, 58, 203-232.

Coccia, M., Bartolini, M., Luzzi, S., Provinciali, L., \& Lambon Ralph, M. A. (2004). Semantic memory is an amodal, dynamic system: Evidence from the interaction of naming and object use in semantic dementia. Cognitive Neuropsychology, 21, 513-527.

Collette, F., Hogge, M., Salmon, E., \& Van der Linden, M. (2006). Exploration of the neural substrate of executive functioning by functional imaging. Neuroscience, 139, 209-221.

Collette, F., Van der Linden, M., Laureys, S., Delfiore, G., Degueldre, C., Luxen, A., et al. (2005). Exploring the unity and diversity of the neural substrates of executive functioning. Human Brain Mapping, 25, 409-423.

Conn, M. (2003). Neuroscience in medicine. Totowa, NJ: Humana Press.

Cristescu, T. C., Devlin, J. T., \& Nobre, A. C. (2006). Orientating attention to semantic categories. Neuroimage, 33, 1178-1187.

Damasio, H., \& Damasio, A. R. (1989). Lesion analysis in neuropsychology. New York: Oxford University Press.

Demb, J. B., Desmond, J. E., Wagner, A. D., Vaidya, C. J., Glover, G. H., \& Gabrieli, J. D. (1995). Semantic encoding and retrieval in the left inferior prefrontal cortex: A functional MRI study of task difficulty and process specificity. Journal of Neuroscience, 15, 5870-5878.

Garavan, H., Ross, T. J., Li, S. J., \& Stein, E. A. (2000). A parametric manipulation of central executive functioning. Cerebral Cortex, 10, 585-592.

Gennari, S. P., MacDonald, M. C., Postle, B. R., \& Seidenberg, M. S. (2007). Context-dependent interpretation of words: Evidence for interactive neural processes. Neuroimage, 35, 1278-1286.

Gloor, P. (1997). The temporal lobe and limbic system. New York: Oxford University Press.

Goodglass, H. (1983). The assessment of aphasia and related disorders (2nd ed.). Philadelphia: Lea \& Febiger.

Gotts, S. J., \& Plaut, D. C. (2002). The impact of synaptic depression following brain damage: A connectionist account of "access/refractory" and "degraded-store" semantic impairments. Cognitive, Affective, and Behavioral Neuroscience, 2, 187-213.

Hart, J., \& Gordon, B. (1990). Delineation of single-word semantic comprehension deficits in aphasia, with anatomical correlation. Annals of Neurology, 27, 226-231.

Hirshorn, E. A., \& Thompson-Schill, S. L. (2006). Role of the left inferior frontal gyrus in covert word retrieval: Neural correlates of switching during verbal fluency. Neuropsychologia, 44, 2547-2557.

Hon, N., Epstein, R. A., Owen, A. M., \& Duncan, J. (2006). Frontoparietal activity with minimal decision and control. Journal of Neuroscience, 26, 9805-9809.

Howard, D., \& Orchard Lisle, V. (1984). On the origins of semantic errors in naming: Evidence from the case of a global aphasic. Cognitive Neuropsychology, 1, 163-190.

Howard, D., \& Patterson, K. (1992). Pyramid and palm trees: A test of semantic access from pictures and words. Bury St. Edmunds: Thames Valley Test Company.

Jefferies, E., Baker, S. S., Doran, M., \& Lambon Ralph, M. A. (2007). Refractory effects in stroke aphasia: A consequence of poor semantic control. Neuropsychologia, 45, 1065-1079.

Jefferies, E., \& Lambon Ralph, M. A. (2006). Semantic impairment in stroke aphasia versus semantic dementia: A case-series comparison. Brain, 129, 2132-2147.

Jefferies, E., Patterson, K., \& Lambon Ralph, M. A. (2008). Deficits of knowledge versus executive control in semantic cognition: Insights from cued naming. Neuropsychologia, 46, 649-658.

Kay, J., Lesser, R., \& Coltheart, M. (1992). Psycholinguistic assessments of language processing in aphasia (PALPA). Hove, UK: Lawrence Erlbaum Associates. 
Koenig, K., \& Grossman, M. (2007). Process and content in semantic memory. In J. Hart \& M. A. Kraut (Eds.), Neural basis of semantic memory. Cambridge: Cambridge University Press.

Lambon Ralph, M. A., \& Patterson, K. (2008). Generalization and differentiation in semantic memory: Insights from semantic dementia. Annals of the New York Academy of Science, 1124, 61-76.

Lambon Ralph, M. A., Sage, K., \& Roberts, J. (2000). Classical anomia: A neuropsychological perspective on speech production. Neuropsychologia, 38, 186-202.

Lee, S. S., \& Dapretto, M. (2006). Metaphorical vs. literal word meanings: fMRI evidence against a selective role of the right hemisphere. Neuroimage, 29, 536-544.

Levelt, W. J. M. (1992). Accessing words in speech production: Stages, processes and representations. Cognition, 42, 1-22.

Luzzi, S., Snowden, J., Neary, D., Coccia, M., Provinciali, L., \& Lambon Ralph, M. A. (2007). Distinct patterns of olfactory impairments in Alzheimer's disease, semantic dementia, frontotemporal dementia and corticobasal degeneration. Neuropsychologia, 45, 1823-1831.

Martin, A. (2008). The representation of object concepts in the brain. Annual Review of Psychology, 58, 25-45.

Milner, B. (1964). Effects of different brain lesions on card sorting: The role of the frontal lobes. Archives of Neurology, 9, 100-110.

Mummery, C. J., Patterson, K., Price, C. J., Ashburner, J., Frackowiak, R. S. J., \& Hodges, J. R. (2000). A voxel-based morphometry study of semantic dementia: Relationship between temporal lobe atrophy and semantic memory. Annals of Neurology, 47, 36-45.

Nagel, I. E., Schumacher, E. H., Goebel, R., \& D’Esposito, M. (2008). Functional MRI investigation of verbal selection mechanisms in lateral prefrontal cortex. Neuroimage, 43, 801-807.

Nestor, P. J., Fryer, T. D., \& Hodges, J. R. (2006). Declarative memory impairments in Alzheimer's disease and semantic dementia. Neuroimage, 30, 1010-1020.

Onifer, W., \& Swinney, D. A. (1981). Accessing lexical ambiguities during sentence comprehension: Effects of frequency of meaning and contextual bias. Memory $\varepsilon$ Cognition, 9, 225-236.

Parker, G. J. M., Luzzi, S., Alexander, D. C., Wheeler-Kingshott, C. A. M., Ciccarelli, O., \& Lambon Ralph, M. A. (2005). Lateralization of ventral and dorsal auditory-language pathways in the human brain. Neuroimage, 24, 656-666.

Patterson, K., Nestor, P. J., \& Rogers, T. (2007). Where do you know what you know? The representation of semantic knowledge in the human brain. Nature Reviews Neuroscience, 8, 976-987.

Peers, P. V., Ludwig, C. J. H., Rorden, C., Cusack, R., Bonfiglioli, C., Bundesen, C., et al. (2005). Attentional functions of parietal and frontal cortex. Cerebral Cortex, 15, 1469-1484.

Raven, J. C. (1962). Coloured progressive matrices sets A $A B, B$. London: $\mathrm{H}$. K. Lewis.

Robertson, I. H., Ward, T., Ridgeway, V., \& Nimmo-Smith, I. (1994). The test of everyday attention. London: Thames Valley Test Company.

Rodd, J. M., Davis, M. H., \& Johnsrude, I. S. (2005). The neural mechanisms of speech comprehension: fMRI studies of semantic ambiguity. Cerebral Cortex, 15, 1261-1269.

Rodd, J. M., Gaskell, M. G., \& Marslen-Wilson, W. D. (2004). Modelling the effects of semantic ambiguity in word recognition. Cognitive Science, 28, 89-104.

Rogers, T. T., Lambon Ralph, M. A., Garrard, P., Bozeat, S., McClelland, J. L., Hodges, J. R., et al. (2004). The structure and deterioration of semantic memory: A neuropsychological and computational investigation. Psychological Review, 111, 205-235.

Rowe, J., Hughes, L., Eckstein, D., \& Owen, A. M. (2008). Rule-selection and action-selection have a shared neuroanatomical basis in the human prefrontal and parietal cortex. Cerebral Cortex, 18, 2275-2285.

Saffran, E. M. (2000). The organisation of semantic memory: In support of a distributed model. Brain and Language, 71, 204-212.

Samson, D., Connolly, C., \& Humphreys, G. W. (2007). When "happy" means "sad": Neuropsychological evidence for the right prefrontal cortex contribution to executive semantic processing. Neuropsychologia, 45, 896-904.

Simpson, G. B. (1985). Lexical ambiguity and its role in models word recognition. Psychological Bulletin, 96, 316-340.

Simpson, G. B., \& Burgess, C. (1984). Activation and selection processes in the recognition of ambiguous words. Journal of Experimental Psychology: Human Perception and Performance, 11, 28-39.

Snodgrass, J. G., \& Vanderwart, M. (1980). A standardized set of 260 pictures: Norms for name agreement, image agreement, familiarity and visual complexity. Journal of Experimental Psychology: Human Learning and Memory, 6, 174-215.

Stuss, D. T., Levine, B., Alexander, M. P., Hong, J., Palumbo, C., \& Hamer, L. (2000). Wisconsin card sorting test performance in patients with focal frontal and posterior brain damage: Effects of lesion location and test structure on separable cognitive processes. Neuropsychologia, 34, 388-402.

Thompson-Schill, S. L., D'Esposito, M., Aguirre, G. K., \& Farah, M. J. (1997). Role of left inferior prefrontal cortex in retrieval of semantic knowledge: A reevaluation. Proceedings of the National Academy of Sciences, U.S.A., 94, 14792-14797.

Twilley, L. C., Dixon, P., Taylor, D., \& Clark, K. (1994). University of Alberta norms of relative meaning frequency for 566 homographs. Memory and Cognition, 22, 111-126.

Vigneau, M., Beaucousin, V., Hervé, P. Y., Duffau, H., Crivello, F., Houdé, O., et al. (2006). Meta-analyzing left hemisphere language areas: Phonology, semantics, and sentence processing. Neuroimage, 30, 1414-1432.

Wagner, A. D., Pare-Blagoev, E. J., Clark, J., \& Poldrack, R. A. (2001). Recovering meaning; Left prefrontal cortex guides controlled semantic retrieval. Neuron, 31, 329-338.

Warrington, E. K., \& Cipolotti, L. (1996). Word comprehension: The distinction between refractory and storage impairments. Brain, 119, 611-625.

Warrington, E. K., \& James, M. (1991). The Visual Object and Space Perception Battery. Bury St. Edmunds: Thames Valley Test Company.

Warrington, E. K., \& McCarthy, R. A. (1983). Category specific access dysphasia. Brain, 106, 859-878.

Warrington, E. K., \& Shallice, T. (1984). Category specific semantic impairments. Brain, 107, 829-853.

Wechsler, D. (1987). Wechsler Memory Scale-Revised (WMS-R). New York: Psychological Corporation.

Wise, R. (2003). Language systems in normal and aphasic human subjects: Functional imaging studies and inferences from animal studies. British Medical Bulletin, 65, 95-119.

Zempleni, M.-Z., Renken, R., Hoeks, J. C. J., Hoogduin, J. M., \& Stowe, L. A. (2007). Semantic ambiguity processing in sentence context: Evidence from event-related fMRI. Neuroimage, 34, 1270-1279. 